\title{
Seguimiento de pacientes con pie diabético en un hospital de alta complejidad del norte del Perú
}

\section{Follow-up of patients with diabetic foot in a highly complex hospital in northern Peru}

\author{
Franco León-Jiménez ${ }^{1,2, a}$, Liliana Torres-Samamé $e^{3, b}$, Lida Altamirano-Cardozo ${ }^{3, c}$, Ana Paula Sofía Navarro-Ríos ${ }^{4, d}$, \\ Giulliana Almendra Meléndez-Ramírez ${ }^{4, e}$ \\ ${ }^{1}$ Centro de Salud Global, Universidad Peruana Cayetano Heredia. Lima, Perú. \\ ${ }^{2}$ Hospital Santa Rosa. Piura, Perú. \\ ${ }^{3}$ Hospital Regional Lambayeque. Lambayeque, Perú. \\ ${ }^{4}$ Universidad Católica Santo Toribio de Mogrovejo. Lambayeque, Perú. \\ ${ }^{a}$ Médico internista, ORCID: https://orcid.org/0000-0002-9418-3236 \\ ${ }^{\mathrm{b}}$ Médico endocrinólogo, ORCID: https://orcid.org/0000-0001-5040-460X \\ ' Licenciada en enfermeria, ORCID: https://orcid.org/0000-0001-7167-8036 \\ ${ }^{\mathrm{d}}$ Interna de medicina, ORCID: https://orcid.org/0000-0002-0214-0061 \\ ${ }^{\mathrm{e}}$ Interna de medicina, ORCID: https://orcid.org/0000-0003-1071-1474
}

An Fac med. 2021;82(2):124-30. / DOI: https://doi.org/10.15381/anales.v82i2.20103.

\begin{abstract}
Correspondencia:
Franco León Jiménez

francoernestole@gmail.com

Recibido: 7 de abril 2021

Aprobado: 10 de agosto 2021

Publicación en línea: 15 de agosto 2021

Conflictos de interés: Los autores

declaran no tener conflictos de interés.

Fuente de financiamiento: Autofinanciado

Citar como: León-Jimenez F, TorresSamamé L, Altamirano-Cardozo L, Navarro-Ríos APS, Meléndez-Ramírez $G A$. Seguimiento de pacientes con pie diabético en un hospital de alta complejidad del norte del Perú. An Fac med. 2021;82(2):124-30.DOI: https://doi. org/10.15381/anales.v82i2.20103.
\end{abstract}

\section{Resumen}

Introducción. El pie diabético es una complicación frecuente de la diabetes. Los datos son escasos en el norte peruano. Objetivo. Describir la evolución clínica del pie diabético en un hospital de LambayequePerú, entre los años 2018 y 2019. Métodos. Estudio descriptivo, prospectivo. Los pacientes fueron captados por emergencia/consulta y seguidos por la unidad de pie diabético mediante visitas diarias y curaciones. Resultados. Seguimiento desde agosto del 2018 a octubre 2019. Hubo 136 pacientes; mediana de edad: 63 años $(\mathrm{RIC}=54-86)$ y 50,38\% fueron hombres. La mediana de años con diabetes y tiempo de hospitalización fue 10 años y 10 dias; $40 \%$ y 21\% tuvo trauma y amputación previa. Las frecuencias de hipertensión arterial, nefropatía diabética, enfermedad renal crónica, síndrome coronario agudo y evento cerebrovascular previo fueron: $52,9 \%, 26,4 \%, 33,3 \%, 2,9 \%$ y $16,6 \%$, respectivamente; $50,7 \%$ tuvo compromiso de pie derecho, $58,1 \%$ del izquierdo y $8,6 \%$, de ambos. El Wagner inicial más frecuente fue IV: $30,16 \%$, seguido de II: $25,40 \%$ y el final más frecuente fue IV: $42,15 \%$ seguido del II: $22,31 \%$. Se amputaron $41,22 \%$, mayores: $28,24 \%$ y menores $11,8 \% ; 25,6 \%$ fueron supracondileas; $39,6 \%$ tuvo sepsis, evolución desfavorable $38,58 \%$ y fallecieron $9,44 \%$. En los amputados, la mediana de días antes de la amputación fue 12. Conclusiones. La frecuencia de amputación y mortalidad fue mayor a la de estudios anteriores. Hubo una alta frecuencia de evolución desfavorable: amputación/muerte o empeoramiento de la gangrena.

Palabras clave: Pie Diabético; Evolución Clínica; Diabetes Mellitus Tipo 2; Perú (fuente: DeCS BIREME).

\section{Abstract}

Introduction. Diabetic foot is a frequent complication of diabetes. Data are scarce in northern Peru. Objective. To describe the clinical evolution of the diabetic foot in a hospital in Lambayeque-Peru, between 2018 and 2019. Methods. Descriptive, prospective study. The patients were recruited by emergency/consultation and followed by the diabetic foot unit through daily visits and dressings. Results. Follow-up from August 2018 to October 2019. There were 136 patients; median age: 63 years $(I Q R=54-86)$ and $50.38 \%$ were men. The median number of years with diabetes and hospitalization time was 10 years and 10 days; $40 \%$ and $21 \%$ had trauma and previous amputation. The frequencies of high blood pressure, diabetic nephropathy, chronic kidney disease, previous acute coronary syndrome and cerebrovascular event were: $52.9 \%, 26.4 \%, 33.3 \%$, $2.9 \%$ and $16.6 \%$, respectively; $50.7 \%$ had involvement of the right foot, $58.1 \%$ of the left and $8.6 \%$ of both. The most frequent initial Wagner was IV: $30.16 \%$, followed by II: $25.40 \%$ and the most frequent final was IV: 42.15\% followed by II: $22.31 \% .41 .22 \%$ were amputated, mayor amputations: $28.24 \%$ and minor amputations $11.8 \% ; 25.6 \%$ were supracondylar; $39.6 \%$ had sepsis, $38.58 \%$ unfavorable evolution and $9.44 \%$ died. In amputees, the median number of days before amputation was 12 . Conclusions. the frequency of amputation and mortality was higher than in previous studies. There was a high frequency of unfavorable evolution: amputation/death or worsening of gangrene.

Keywords: Diabetic Foot; Prospective Study; Diabetes Mellitus, Type 2; Peru (source: MeSH NLM). 


\section{INTRODUCCIÓN}

El pie diabético (PD) es una complicación frecuente en el curso de la diabetes. En una reciente revisión sistemática, la prevalencia mundial fue de 6,3\% (IC95\%: $5,4-7,3 \%)^{(1)}$. Se asocia a ingresos frecuentes a emergencia ${ }^{(2)}$, costos elevados al sistema de salud ${ }^{(3)}$, deficiente calidad de vida ${ }^{(4)}$ y es la primera causa de amputaciones del miembro inferior ${ }^{(5)}$. Según el estudio situación de la vigilancia epidemiológica de diabetes en establecimientos de salud del Perú del primer trimestre del 2019, en 3920 diabéticos de hospitales del Perú, sólo 35,6\% tenían buen control metabólico, 19,5\% tenían neuropatía periférica y $7,7 \%$, pie diabético ${ }^{(6)}$. En el Hospital Regional Lambayeque se halló que del total de pacientes diabéticos registrados, 25/145 $(17,2 \%)$ tuvieron PD; de estos, 9,72\% tenían al menos un miembro amputado ${ }^{(7)}$.

Recientemente, Yovera-Aldana en un estudio multicéntrico peruano en 8346 pacientes con diabetes de 39 hospitales, halló una prevalencia puntual de PD de $18,9 \%$ (IC $95 \% 16,7-21,1)$ y específicamente en Lambayeque de $15,2(5,1-31,9)^{(8)}$.

En el estudio Eurodiale, estudio prospectivo, observacional en 14 países europeos en 1232 pacientes con úlcera por pie diabético (UPD), entre el 2003 y el 2004, los puntos finales al año de seguimiento fueron: cicatrización de la úlcera, amputación mayor y muerte. Al año, 23\% no habían cicatrizado, $12 \%$ aún se encontraban en tratamiento, $5 \%$ se habían amputado y $6 \%$ fallecieron. Hubo mayor frecuencia de amputación, muerte y menor cicatrización de úlcera en quienes tenían insuficiencia vascular periférica. Los factores asociados a no cicatrización fueron: ser varón, edad mayor de 65 años, insuficiencia cardiaca, enfermedad renal crónica, inhabilidad para pararse o caminar sin ayuda, gran tamaño de la úlcera, neuropatía periférica e insuficiencia vascular periférica ${ }^{(2)}$.

Por otro lado, la presencia de un equipo multidisciplinario de profesionales a cargo de los pacientes con PD (unidades de PD), ha demostrado mejorar la sobrevida y disminuir las amputaciones. Krishnan en Ipswich, Inglaterra, entre 1995 y el 2005, halló que la presencia de una unidad de PD, podía disminuir la incidencia de amputaciones mayores en $62 \%$ y amputaciones totales en $40 \%{ }^{(9)}$. Por su parte, Wang, en un análisis retrospectivo de 648 pacientes desde el 2004 al 2013, halló que la frecuencia de amputaciones mayores disminuyó de 9,5\% en 2004 y $14,5 \%$ en el 2005 a menos de $5 \%$ luego del 2006, en especial en pacientes con Wagner III y IV. Durante este tiempo en esta unidad, se iniciaron las actividades de un equipo multidisciplinario liderados por un endocrinólogo y un podiatra ${ }^{(10)}$.

Una alternativa que podría mejorar la gestión y el seguimiento de los pacientes con descongestionamiento de las emergencias y de los consultorios de PD es la evolución a distancia de la progresión de las lesiones mediante fotografías tomadas por los mismos pacientes y/o sus cuidadores mediante el celular, permitiendo un manejo ambulatorio en aquellos pacientes sin criterios de sepsis ni de amputación. Ello ha sido evaluado en varios escenarios, con resultados a favor del uso de esta estrategia. La sobrevida, amputaciones y tiempo de cicatrización de la lesión no diferiría al compararlo con la vista programada del paciente ${ }^{(11-14)}$. La gestión de las citas sería más eficiente. Asimismo, los mensajes recordatorios sobre el cuidado del pie, el control de la glicemia y hemoglobina glicosilada, así como recordatorios de citas, el envío de mensajes positivos y alentadores a los pacientes, pueden potenciarse mediante el uso de estos dispositivos ${ }^{(13,14)}$.

Al ser el PD un problema de salud pública ${ }^{(1)}$, con ingresos frecuentes a los servicios de emergencia ${ }^{(2)}$, costos elevados al sistema de salud ${ }^{(3)}$, siendo la prevalencia puntual en un hospital III de Lambayeque de $15,2 \%{ }^{(8)}$ y ante la ausencia de data reciente en el norte peruano sobre la caracterización y evolución clínica, se sustentó la necesidad de realizar el presente estudio. Los resultados pueden servir a los decisores de salud para mejorar la gestión en la estrategia de enfermedades crónicas no transmisibles. Así, el objetivo del estudio fue describir la evolución clínica del pie diabético en un hospital de alta complejidad del norte del Perú.

\section{MÉTODOS}

\section{Tipo de estudio}

Se realizó un estudio observacional, descriptivo y prospectivo

\section{Población y muestra}

Cohorte única sin grupo de comparación, de todos los pacientes que ingresaron con diagnóstico de pie diabético por emergencia o consulta externa del Hospital Regional Lambayeque del Ministerio de Salud del Perú, captados por el investigador principal y seguidos diariamente en emergencia y en el servicio de medicina, hasta la resolución del cuadro: alta (luego de manejo conservador), amputación o fallecimiento. Todos los pacientes fueron diabéticos tipo 2 .

\section{Manejo y seguimiento de pacientes con pie diabético}

Los manejos propuestos para el manejo de pie diabético fueron, Wagner II: antibioticoterapia de amplio espectro y limpieza con solución fisiológica al 0,9\%; Wagner III: hospitalización, antibioticoterapia de amplio espectro, manejo de sepsis, anestesia local y drenaje descompresivo de absceso e interconsulta a traumatología para curetaje si había osteomielitis en radiografía; Wagner IV: hospitalización, antibioticoterapia de amplio espectro, manejo de sepsis y evaluación por traumatología para posibilidades de amputación y Wagner V: manejo previo y amputación. La curación de los pacientes con Wagner III, IV y V se realizó de manera diaria por uno de los miembros del equipo, mediante anestesia local, lavado con solución fisiológica y drenaje de abscesos localizados.

Se creó un sistema de alerta por el cual, uno de los investigadores abordaba al paciente $\mathrm{y} / \mathrm{o}$ familiar $\mathrm{y}$ tras autorización, tomaba 2 fotos de las lesiones, que, en ese momento, eran compartidas por aplicativo WhatsApp al grupo de la Unidad de PD del Hospital Regional Lambayeque. Tras lectura de los comentarios de las diferentes especialidades (endocrinología, cirugía cardiovascular, traumatología), se dejaba constancia en la historia clínica de la opinión del grupo sobre el manejo propuesto. La extensión, profundidad, presencia de secreción y compromiso de tendones fueron características 
evaluadas por este registro. Se describieron aspectos clínicos, de laboratorio y ecográficos; se usó la clasificación Wagner para la clasificación inicial y final de la lesión: Wagner $0=$ pie en riesgo, Wagner I= úlcera superficial, Wagner II= infección y úlcera profunda hasta ligamentos, Wagner III= osteomielitis, Wagner IV= necrosis parcial del pie; Wagner $V=$ necrosis total del pie ${ }^{(15)}$. Se consideró una evolución desfavorable a la muerte o amputación mayor, hasta el final del seguimiento.

Por otro lado, las fotos eran ubicadas en un archivo en Google Drive, en la cuenta del investigador principal, para determinar la evolución. Según roles establecidos, un miembro de la Unidad PD realizaba curaciones/drenaje/limpieza y toma diaria de fotografías de las lesiones y hacía seguimiento diario de los exámenes de ayuda diagnóstica (ecografía doppler, radiografías del pie, examenes de laboratorio). Al finalizar el día, se socializaba este reporte y se discutían los avances. También, la gestión de las imágenes se realizó usando la aplicación Carestream ${ }^{(16)}$ para la descarga, visualización, evaluación y almacenamiento de las radiografías. Se obtuvieron datos de las historias clínicas. Al alta, los pacientes eran citados al consultorio de PD y evaluados según la evolución y severidad de las lesiones.

\section{Aspectos éticos}

El estudio tuvo la aprobación del Comité de Ética en Investigación del Hospital Regional Lambayeque. A los pacientes se les consultó para participar en el estudio, revisar su historia clínica y tomar fotografías. Se usó un consentimiento informado en el cual se incluía la confidencialidad de la información y el uso del material (incluyendo las fotos) sólo para los fines del estudio. Al archivo Google Drive sólo tuvo acceso el investigador principal. Luego de cada visita se informó al paciente o su familiar sobre la evolución de la lesión y la conducta terapéutica a seguir.

\section{RESULTADOS}

El seguimiento fue desde agosto del 2018 a octubre 2019. Hubo136 pacientes con PD. La mediana de edad fue 63 años (RIC = 54-86) y 67/133 (50,38\%) fueron hombres. La mediana de años con diabetes y tiempo de hospitalización fueron 10 años y 10 días, respectivamente. Otros tiempos de atención de los pacientes y algunas características generales figuran en la tabla 1; $40 \%$ refirieron trauma previo en el pie y $21 \%$ amputación previa en al menos uno de los pies. Las frecuencias de hipertensión arterial, nefropatía diabética, enfermedad renal crónica, síndrome coronario agudo previo y evento cerebrovascular previo fueron: 54/102 (52,94\%), 27/102 (26,47\%), $34 / 102$ (33,33\%), 3/102 (2,9\%) y $17 / 102$ $(16,67 \%)$, respectivamente.

Tuvieron compromiso de pie derecho $65 / 128$ (50,78\%), del izquierdo 75/129 (58,13\%), y $11 / 128$ (8,59\%) de ambos; $24 / 130(18,46 \%)$ tuvieron dos episodios de PD y $3 / 130$ (2,31\%), tres, durante toda su vida. En 6 pacientes no se obtuvo el dato del número de episodios. El Wagner inicial más frecuente fue IV: 30,16\%, seguido de II: 25,40\%. El Wagner final más frecuente fue IV: $42,15 \%$ seguido del II: 22,31\%. La distribución total del Wagner al inicio y al final del seguimiento se muestra en la tabla 2.

Sólo se pudo obtener fotos en 107/131 pacientes: $81,67 \%$. Tuvieron cetoacidosis diabética $7 / 124$ (5,64\%). Hubo una gestante y un paciente con VIH-SIDA; un paciente fue a revascularización.
Hubo una frecuencia de 54/131 (41,22\%) de amputaciones. Amputaciones mayores: $37 / 131(28,24 \%)$ y amputaciones menores 16/125 (11,8\%); en $1 / 131(0,76 \%)$ no se tuvo el dato si fue mayor o menor. Hubo 32/125 (25,6\%) amputaciones supracondíleas. La amputación menor más frecuente fue en los dedos: 12/125 (9,6\%). Tuvieron sepsis: $48 / 121(39,66 \%)$ y la evolución fue desfavorable en: $49 / 127$ (38,58\%); fallecieron $12 / 127$ (9,44\%). En los amputados, la mediana de días antes de la amputación fue 12 días.

Tal como figura en la tabla 3, sólo se hizo ecografía doppler en 90/136 pacientes $(66,1 \%)$; en estos, 83 (91,2\%) tuvieron insuficiencia venosa periferica, siendo mayor de un año en 71,6\%. En la tabla 3 se muestra las medianas de glicemia y hemoglobina glicosilada.

Por otro lado, usaban insulina 42/102 (41,18\%), antidiabéticos orales 43/102 $(42,16 \%)$ y ambos tratamientos: $19 / 102$ $(18,81 \%)$.

En relación a datos de gestión, 87/102 $(85,29 \%)$ fueron evaluados por la estrategia de enfermedades crónicas no transmisibles, 23/102 (22,55\%) por cirugía cardiovascular y 43/102 (42,16\%) por traumatología. Sólo 38,23\% de pacientes contaban con una radiografía de pie en el sistema de gestión de imágenes.

Tabla 1. Características del tiempo de enfermedad, hospitalización y antecedentes de pacientes con pie diabético atendidos en el Hospital Regional de Lambayeque entre los años 2018 y 2019.

\begin{tabular}{lcc} 
Características & Mediana & RIC \\
\hline Tiempo hospitalización (días) ( $\mathrm{n}=50)$ & 10 & $4-45$ \\
\hline Tiempo de diagnóstico de diabetes mellitus tipo 2 (meses) $(\mathrm{n}=109)$ & 120 & $64-396$ \\
\hline Tiempo enfermedad (días) ( $\mathrm{n}=109)$ & 6 & $3-90$ \\
\hline & $\mathrm{N}$ & $\%$ \\
\hline Frecuencia de hospitalización ( $\mathrm{n}=128)$ & 79 & 61,72 \\
\hline Antecedente de amputación previa $(\mathrm{n}=130)$ & 28 & 21,37 \\
\hline Antecedente de trauma $(\mathrm{n}=103)$ & 41 & 39,81 \\
\hline Número de veces en emergencia $(\mathrm{n}=131)$ & & \\
\hline Ninguna & 35 & 26,72 \\
\hline 1 Vez & 88 & 67,18 \\
\hline Dos Veces & 7 & 5,34 \\
\hline Tres veces & 1 & 0,76 \\
\hline Frecuencia de toma de fotos $(\mathrm{n}=131)$ & 107 & 81,68 \\
\hline Retiro Voluntario $(\mathrm{n}=59)$ & 14 & 23,72 \\
\hline
\end{tabular}


Tabla 2. Características clínicas de pacientes con pie diabético atendidos en el Hospital Regional de Lambayeque entre los años 2018 y 2019

\begin{tabular}{lcc} 
Compromiso pie izquierdo (n=75/129) & & $\%$ \\
\hline Dedos & 37 & 49,33 \\
\hline Calcáneo & 16 & 21,33 \\
\hline Dorso de pie & 6 & 8,0 \\
\hline Otros & 6 & 8,0 \\
\hline Calcáneo y dedos & 5 & 6,66 \\
\hline Planta del pie & 3 & 4,00 \\
\hline Compromiso pie derecho (n=66/129) & & $\mathbf{2 1 , 2 1}$ \\
\hline Calcáneo & 14 & 39,39 \\
\hline Dedos & 26 & 10,6 \\
\hline Otros & 7 & 9,09 \\
\hline Planta del pie & 6 & 6,06 \\
\hline Dorso de pie & 4 & 4,5 \\
\hline Calcáneo y dedos & 3 & 4,5 \\
\hline Calcáneo y dorso & 3 & 3,03 \\
\hline Borde externo pie & 2 & $\%$ \\
\hline Wagner Inicial (n= 126) & & 4
\end{tabular}

\section{Wagner Inicial $(n=126)$}

\begin{tabular}{ccc}
0 & 6 & 4,76 \\
\hline 1 & 22 & 17,46 \\
\hline 2 & 32 & 25,40 \\
\hline 3 & 21 & 16,67 \\
\hline 4 & 38 & 30,16 \\
\hline 5 & 7 & 5,56
\end{tabular}

Wagner Final ( $\mathrm{n}=121)$

$\%$

\begin{tabular}{ccc}
0 & 5 & 4,13 \\
\hline 1 & 19 & 15,7 \\
\hline 2 & 27 & 22,31 \\
\hline 3 & 12 & 9,92 \\
\hline 4 & 51 & 42,15 \\
\hline 5 & 7 & 5,79 \\
Amputación $(\mathbf{n}=\mathbf{1 3 1})$ & & $\%$
\end{tabular}

\begin{tabular}{|c|c|c|}
\hline No & 86 & 63,7 \\
\hline $\mathrm{Si}$ & 54 & 41,22 \\
\hline Número de amputaciones ( $n=132$ ) & & $\%$ \\
\hline Ninguna & 80 & 61,48 \\
\hline Una & 47 & 34,81 \\
\hline Dos & 5 & 3,70 \\
\hline Tipo de amputación pie I ( $n=22$ ) & & $\%$ \\
\hline Supracondílea & 9 & 40,9 \\
\hline Dedos & 8 & 36,4 \\
\hline Infracondílea & 3 & 13,6 \\
\hline Tarso & 2 & 9,09 \\
\hline Tipo de amputación pie $D(n=30)$ & & $\%$ \\
\hline Supracondílea & 23 & 76,7 \\
\hline Dedos & 4 & 16,7 \\
\hline Infracondílea & 2 & 13,6 \\
\hline Sepsis (n=121) & 48 & 36,7 \\
\hline Fallecimiento ( $n=127$ ) & 12 & 9,4 \\
\hline
\end{tabular}

\section{DISCUSIÓN}

La revisión sistemática (RS) de 67 estudios y 800000 pacientes hecha por Zhang en el 2017, halló que la UPD tiene una prevalencia en hombres de 4,5\% (IC 95\%: 3,7 - 5,2\%) y de 3,5\% (IC 95\%: 2,8-4,2\%) en mujeres ${ }^{(1)}$. En el estudio Eurodiale, 64,6\% fueron varones ${ }^{(2)}$. Estas cifras pueden ser distintas a nuestro estudio en el que hubo igual porcentaje de ambos sexos. En ambos estudios no participó ningún país sudamericano y la enfermedad puede ser diferente en nuestra población; esto es materia de investigación. Yovera-Aldana en el estudio multicéntrico realizado en 39 hospitales peruanos halló una frecuencia de sexo masculino de $73 \%{ }^{(8)}$, mayor a nuestro estudio.

En relación al tiempo de enfermedad, se registró el tiempo en el cual el paciente observó una complicación de una lesión que probablemente ya tenía desde antes y no el tiempo de una UPD. Esto puede explicar la diferencia del tiempo: 12 días en nuestro estudio y en la RS de Zang, 58\% tenían UPD entre 1semana y 3 meses. Ugwu en 6 hospitales de alta complejidad en el África, halló que la duración de una úlcera más de un mes (OR 10,3, 95\% IC 4-26,1), se asoció a amputación. En nuestro estudio, la mediana del tiempo de enfermedad previo a ingreso fue de 6 días. Este es un sesgo pues probablemente los pacientes sólo reporten el tiempo de la complicación de una UPD ${ }^{(18)}$.

En relación al tiempo con diabetes, se halló que la mediana fue de 10 años, similar a lo hallado en la RS de Zhang: 69,8\% referían tener más de 10 años con la enfermedad (1). Yovera-Aldana halló una media de 15 años con diabetes en sus pacientes ${ }^{(8)}$.

La alta frecuencia de amputación mayor (28\%) fue muy superior a la de otros estudios: Yiang en China $(5,1 \%)^{(17)}$, Eurodiale (5\%) ${ }^{(2)}$ y menor al 35,4\% del estudio nigeriano de Ugwu ${ }^{(18)}$. En el estudio de Yiang y en el Eurodiale, al inicio sólo se incluyeron pacientes con UPD, a diferencia de nuestro estudio en el que más del 50\% de pacientes tenían al ingreso un Wagner de III a más. Además, en el segundo se excluyeron aquellos con tiempo de vida 
Tabla 3. Ecografía doppler y examenes de laboratorio de pacientes con pie diabético atendidos en el Hospital Regional de Lambayeque entre los años 2018 y 2019.

\begin{tabular}{lcc} 
Se hizo Ecodoppler $(\mathbf{n = 1 3 6 )}$ & $\mathbf{N}$ & $\mathbf{\%}$ \\
\hline Sí & 90 & 66,18 \\
\hline No & 28 & 20,59 \\
\hline Desconocido & 18 & 13,24 \\
\hline IVP por Ecografía Doppler (n=91) & 83 & 91,2 \\
\hline Años con IVP (n=85) & 31 & 28,4 \\
\hline$\leq 1$ año & 54 & 71,6 \\
\hline$>1$ año & $\mathbf{M e d i a n a}$ & $\mathbf{R I C}$ \\
\hline Glicemia en ayunas $(\mathrm{n}=96)$ & $152,5 \mathrm{gr} / \mathrm{dl}$ & $130-292$ \\
\hline HbA1C (n=61) & $7,5 \mathrm{gr} / \mathrm{dl}$ & $6,8-12,4$ \\
\hline Proteinuria (n=19) & $1,220 \mathrm{gr} / 24 \mathrm{~h}$ & $452-3,030$ \\
\hline Creatinina (n=82) & $0,92 \mathrm{gr} / \mathrm{dl}$ & $0,7-8,4$ \\
\hline
\end{tabular}

IVP: insuficiencia venosa periferica

menor de un año y aquellos con úlcera previa ipsilateral. En el estudio de Ugwu en 6 hospitales de alta complejidad, $79,2 \%$ de los pacientes tuvo un Wagner de III a más. Esta característica, un mayor tiempo de seguimiento y las características de ser un país con limitaciones económicas como el nuestro, podrían explicar la similar cifra. Además, en los tres estudios, los pacientes fueron seguidos por un año. En nuestro estudio, en el grupo amputados, la mediana de seguimiento sólo fue 12 días. No se cuenta con el dato del tiempo de seguimiento del total de pacientes

En relación a los factores asociados a amputación en pacientes con PD infectado, Pinar en una RS de 25 estudios observacionales en los que participaron 1873 pacientes que finalmente fueron amputados, halló que, de mayor a menor, los factores asociados a amputación fueron: presencia de gangrena/necrosis del pie $(\mathrm{OR}=9,9)$, Wagner $4 / 5(\mathrm{ORs}=4,3 / 6,4)$, osteomielitis $(O R=4,5)$, infección severa $(O R=3,12)$ y enfermedad vascular periférica $(O R=2,35)$, entre otros ${ }^{(19)}$. En nuestro estudio, todos estos factores fueron frecuentes. Cabe mencionar que el registro de "sepsis" en sólo uno de cada tres de nuestros pacientes, debe ser un subregistro, dada la severidad del cuadro.
También es de notar la baja frecuencia de registro de radiografías de pie en el sistema de imágenes para hacer el diagnóstico inicial de osteomielitis. Este es un proceso que debe mejorarse.

En relación a mortalidad, uno de cada diez pacientes falleció. Esta cifra es parecida a la de un estudio previo del 2016, en pacientes de medicina interna de la emergencia del Hospital Regional de Lambayeque, en el cual 11,7\% fallecieron ${ }^{(20)}$. La pluripatología de estos pacientes podría explicar esta alta frecuencia. Esta cifra es mayor a la reportada en los estudios Eurodiale y a la RS de Zhang ${ }^{(1)}$. Los factores de severidad mencionados líneas arribas podrían contribuir a esta elevada mortalidad.

La presencia de insuficiencia vascular periférica (IVP) es determinante. Zhang halló que estaba presente en el 47,5\% de los pacientes con UPD y que hubo mayor amputación, muerte y menor cicatrización de úlcera en quienes tenían IVP. Ugwu halló que tener IVP tuvo un OR de 2,8 (IC 95\% IC=1,5-5,1) para amputación ${ }^{(18)}$. Lamentablemente, en nuestro estudio sólo se realizó ecogrfía doppler a $66,1 \%$ del total de pacientes. Sin embargo, en este grupo, en más del $90 \%$, se halló IVP. En el estudio nacional de Yovera-Aldana, se encontró que la IVP estaba presente en $68 \%$ del total de pacientes ${ }^{(8)}$. Motivos administrativos como carencia de recursos humanos y demanda de pacientes, explican esta realidad ${ }^{(20)}$. Esta es una tarea pendiente de la estrategia de enfermedades crónicas no transmisibles para un proceso de mejora continua.

Debemos reconocer heterogeneidad en relación a la evaluación de las lesiones. Se tiene la descripción del tipo de lesión por clasificación Wagner y la foto de $85 \%$ de los pacientes, que fue compartida entre los miembros de la unidad de pie diabetico, día a día. Se usó Wagner pues según la RS de Jalilian, es la más usada (5 de 7 estudios) ${ }^{(15)}$. Sin embargo, actualmente, el International Working Group on the Diabetic Foot (IWGDF), ha descrito que la clasificación ideal para comunicación entre evaluadores del PD debería ser el sistema SINBAD (Sitio, Isquemia, Neuropatía, Infección bacteriana y Profundidad) ${ }^{(21)}$. Esta es una limitación de nuestro estudio. La clasificación Wagner no toma en cuenta la presencia de insuficiencia vascular periférica, factor determinante en las complicaciones del PD ${ }^{(19)}$. Por otro lado, Bravo en una cohorte de 250 pacientes con UPD, en el 2018, halló un kappa moderado de concordancia entre observadores de 0,55 (IC 95\%= 0,507-0,593), en relación al Wagner ${ }^{(22)}$.

Cabe mencionar, que no se determinó un kappa previo interobservadores. Existen alternativas como la ofrecida por Wang en Massachusetts en el 2015, quien validó un puntaje para evidenciar el grado de compromiso de la lesión en la que, tras uso de algoritmos, se determinaba el grado de compromiso del PD. El kappa en este estudio fue de 0,4-0,8 (11). Por ello, creemos que es una tarea pendiente generar un índice Kappa entre los observadores de la unidad de pie diabético para hacer confiable la evaluación de lesiones.

Tampoco tenemos el dato de los pacientes que durante el tiempo de seguimiento recayeron. A diferencia del estudio Eurodiale, el más grande prospectivo, en el que se realizó un seguimiento de la UPD por lo menos por un año, en nuestro estudio sólo se evaluó al paciente hasta su desenlace final a corto plazo y los datos de su seguimiento por consulta externa son escaso ${ }^{(2)}$. 
A pesar del esfuerzo realizado, nuestros hallazgos sugieren que deben implementarse medidas más estandarizadas y debe haber un mayor compromiso institucional para el control, seguimiento y gestión de estos pacientes. La frecuencia de retiros voluntarios fue elevada (1 de cada 4). No se han hallado estudios previos en los que se mida esta variable. Ello podría deberse a la negativa de los pacientes a la amputación o al tiempo invertido a la espera de procedimientos como la ecografía doppler, que en la mayoría de casos debía hacerse particularmente por problemas en gestión de turnos. En 1 de cada 5 pacientes por distintos motivos, no se logró tomar una ecografía doppler.

Las unidades de PD han demostrado disminuir la incidencia de amputaciones en el tiempo. Krisnnan, en una población europea, encontró que la presencia de una unidad de profesionales podía disminuir la incidencia de amputaciones mayores en $62 \%$ y amputaciones totales en $40 \%{ }^{(9)}$. Wang, en un análisis retrospectivo de 648 pacientes desde el 2004 al 2013, halló que la frecuencia de amputaciones mayores disminuyó de 9,5\% en 2004 y $14,5 \%$ en el 2005 , a menos de $5 \%$ luego del 2006, en especial en pacientes con Wagner III y IV ${ }^{(10)}$. Por otro lado, las amputaciones aumentan la mortalidad a corto y mediano plazo. Karam analizó la data de los resultados de amputaciones supra e infracondíleas en 6839 pacientes a quienes se les realizó 4001 amputaciones; halló que $10 \%$ de los que son amputados por PD mueren a los 30 días ${ }^{(23)}$. Es necesario mencionar que, a diferencia de los datos de estudios previos, en nuestro estudio no se pudo corroborar una disminución de las complicaciones y de la mortalidad a pesar de la presencia de la unidad de PD. Los pacientes no fueron seguidos tiempo suficiente para hacer esta comparación y esta es otra de las tareas pendientes de la unidad.

La elevada frecuencia de hipertensión arterial $(52,9 \%)$, enfermedad renal crónica $(33,3 \%)$ y accidente vascular cerebral previo $(16,7 \%)$ definen una población con varias comorbilidades, que ensombrecen su pronóstico con riesgo de muerte $y$ amputación mayor. En el estudio multicéntrico peruano, $55 \%$ de los pacientes tenían hipertensión arterial, 42\% enfer- medad renal crónica y sólo 5,7\%, evento vascular previo ${ }^{(8)}$.

Otras limitaciones de nuestro estudio fueron los registros faltantes y datos de algunas variables en algunas observaciones, la elevada congestión en el servicio de emergencia que dificulta los procesos de curación, la deficiencia en el registro en las historias clínicas, la ausencia de datos de laboratorio, ausencia de turnos de ecografía doppler ${ }^{20)}$, la apreciación y consenso clínico mediante fotos compartidas por aplicativo WhatsApp que dependen de la resolución el equipo y la distancia a la cual fueron tomadas las fotos así como que no se lograron fotos de las evoluciones de todos los pacientes.

Llama la atención la baja frecuencia de evaluación de los pacientes por traumatología (42\%) y cirugía vascular (22\%). Probablemente este sea un sesgo de información o subregistro de la actividad de estas especialidades en el servicio de emergencia. Debemos señalar, que, a pesar de no haberse medido la frecuencia de miembros inferiores potencialmente salvados por la presencia de la unidad de PD, esta es una situación en la que un equipo multidisciplinario puede lograr impacto. Sin embargo, para la observación de diferencias se necesitan años de trabajo, como lo observó Krisnnan en Inglaterra ${ }^{(9)}$.

Finalmente, debemos señalar que a pesar de las limitaciones halladas, la alta frecuencia de mortalidad, amputaciones mayores y retiros voluntarios observados, la presencia de una unidad de PD, conformada por distintas especialidades constituye una alternativa atractiva $y$ que ha demostrado, en otros escenarios, disminuir las amputaciones y brindar un mejor soporte al paciente con PD ${ }^{(9,10)}$

En conclusión, según nuestros hallazgos, la frecuencia de amputación y mortalidad fue mayor a la de estudios previos. Hubo una alta frecuencia de evolución desfavorable: amputación, muerte o empeoramiento de la gangrena.

\section{REFERENCIAS BIBLIOGRÁFICAS}

1. Zhang $P$, Lu J, Jing $Y$, Tang S, Zhu D, Bi Y. Global epidemiology of diabetic foot ulce- ration: a systematic review and meta-analysis $^{\dagger}$. Ann Med. 2017 Mar;49(2):106-116. DOI: 10.1080/07853890.2016.1231932

2. Prompers L, Schaper N, Apelqvist J, Edmonds M, Jude E, Mauricio D, et al. Prediction of outcome in individuals with diabetic foot ulcers: focus on the differences between individuals with and without peripheral arterial disease. The EURODIALE Study. Diabetologia. 2008 May;51(5):747-55. DOI: 10.1007/ s00125-008-0940-0

3. Shearer A, Scuffham P, Gordois A, Oglesby A. Predicted costs and outcomes from reduced vibration detection in people with diabetes in the U.S. Diabetes Care. 2003 Aug;26(8):2305-10. DOI: 10.2337/diacare.26.8.2305

4. Boulton AJ, Vileikyte L, Ragnarson-Tennvall G, Apelqvist J. The global burden of diabetic foot disease. Lancet. 2005 Nov 12;366(9498):1719-24. DOI: 10.1016/S0140-6736(05)67698-2

5. Villena JE. Diabetes Mellitus in Peru. Ann Glob Health. 2015 Nov-Dec;81(6):765-75. DOI: 10.1016/j. aogh.2015.12.018

6. Ministerio de Salud del Perú [Internet]. Centro Nacional de Epidemiología, Prevención y Control de Enfermedades. Boletín epidemiológico del Perú. Volumen 28 Semana 14. Disponible en: https:// www.dge.gob.pe/portal/docs/vigilancia/boletines/2019/14.pdf. Fecha de acceso: 16 de marzo 2020.

7. Capitán de la Cruz, Victoria Alejandra. Perfil clínico y laboratorial de los pacientes con enfermedad cardiovascular de un hospital de alta complejidad Lambayeque 2014-2015: informe preliminar (Tesis de bachiller). Disponible en: http://tesis.usat.edu. pe/bitstream/20.500.12423/1727/1/TL_CapitanDeLaCruzVictoria.pdf. Fecha de acceso: 16 de Marzo 2020.

8. Yovera-Aldana M, Sáenz-Bustamante S, QuispeLandeo Y, Agüero-Zamora R, Salcedo J, Sarria C, et al. Nationwide prevalence and clinical characteristics of inpatient diabetic foot complications: A Peruvian multicenter study. Prim Care Diabetes. 2021 Jun;15(3):480-487. DOI: 10.1016/j.pcd.2021.02.009

9. Krishnan S, Nash F, Baker N, Fowler D, Rayman G. Reduction in diabetic amputations over 11 years in a defined U.K. population: benefits of multidisciplinary team work and continuous prospective audit. Diabetes Care. 2008 Jan;31(1):99-101. DOI: 10.2337/ dc07-1178

10. Wang C, Mai L, Yang C, Liu D, Sun K, Song W, et al. Reducing major lower extremity amputations after the introduction of a multidisciplinary team in patient with diabetes foot ulcer. BMC Endocr Disord. 2016 Jul 7;16(1):38. DOI: 10.1186/s12902-016-0111-0

11. Wang L, Pedersen PC, Strong DM, Tulu B, Agu E, Ignotz R, et al. An Automatic Assessment System of Diabetic Foot Ulcers Based on Wound Area Determination, Color Segmentation, and Healing Score Evaluation. J Diabetes Sci Technol. 2015 Aug 7;10(2):421-8. DOI: 10.1177/1932296815599004

12. Kolltveit BH, Gjengedal E, Graue M, Iversen MM, Thorne S, Kirkevold M. Conditions for success in introducing telemedicine in diabetes foot care: a qualitative inquiry. BMC Nurs. 2017 Jan 13;16:2. DOI: 10.1186/s12912-017-0201-y

13. Smith-Strøm H, Igland J, Østbye T, Tell GS, Hausken MF, Graue M, et al. The Effect of Telemedicine Follow-up Care on Diabetes-Related Foot Ulcers: A Cluster-Randomized Controlled Noninferiority Trial. Diabetes Care. 2018 Jan;41(1):96-103. DOI: 10.2337/dc17-1025 
14. Chavez S, Fedele D, Guo Y, Bernier A, Smith M, Warnick J, et al. Mobile Apps for the Management of Diabetes. Diabetes Care. 2017 Oct;40(10):e145e146. DOI: 10.2337/dc17-0853

15. Jalilian M, Ahmadi Sarbarzeh P, Oubari S. Factors Related to Severity of Diabetic Foot Ulcer: A Systematic Review. Diabetes Metab Syndr Obes. 2020 May 25;13:1835-1842. DOI: 10.2147/DMSO.S256243

16. Carestream [Internet]. Disponible en: https:// www.carestream.com/es/es/medical/products/ radiography/carestream-radiography-software/ carestream-image-suite-v4. Fecha de acceso: 29 de Octubre 2019

17. Jiang Y, Wang X, Xia L, Fu X, Xu Z, Ran X, et al. A cohort study of diabetic patients and diabetic foot ulceration patients in China. Wound Repair Regen. 2015 Mar-Apr;23(2):222-30. DOI: 10.1111/wrr.12263
18. Ugwu E, Adeleye O, Gezawa I, Okpe I, Enamino M, Ezeani I. Predictors of lower extremity amputation in patients with diabetic foot ulcer: findings from MEDFUN, a multi-center observational study. J Foot Ankle Res. 2019 Jun 14;12:34. DOI: 10.1186/ s13047-019-0345-y

19. Sen P, Demirdal T, Emir B. Meta-analysis of risk factors for amputation in diabetic foot infections. Diabetes Metab Res Rev. 2019 Oct;35(7):e3165. DOI: 10.1002/dmrr.3165

20. León-Jiménez F, Núñez-Corrales D, MedinaArboleda E, Briones-Díaz E. Tiempos de atención y gestión de datos en la emergencia de un hospital nivel III, Lambayeque-Perú, 2016. Revista Experiencia En Medicina Del Hospital Regional Lambayeque. 2019;5(2): 63-69. DOI: https://doi. org/10.37065/rem.v5i2.337
21. Monteiro-Soares M, Russell D, Boyko EJ, Jeffcoate W, Mills JL, Morbach S, et al. Guidelines on the classification of diabetic foot ulcers (IWGDF 2019). Diabetes Metab Res Rev. 2020 Mar;36 Suppl 1:e3273. DOI: 10.1002/dmrr.3273

22. Bravo-Molina A, Linares-Palomino JP, Vera-Arroyo B, Salmerón-Febres LM, Ros-Die E. Inter-observer agreement of the Wagner, University of Texas and PEDIS classification systems for the diabetic foo syndrome. Foot Ankle Surg. 2018 Feb;24(1):60-64. DOI: 10.1016/j.fas.2016.10.009

23. Karam J, Shepard A, Rubinfeld I. Predictors of operative mortality following major lower extremity amputations using the National Surgical Quality Improvement Program public use data. J Vasc Surg. 2013 Nov;58(5):1276-82. DOI: 10.1016/j. jvs.2013.05.026 